\title{
Reduction in reproductive lifespan of tissue inhibitor of metalloproteinase 1 (TIMP-1)-deficient female mice
}

\author{
W. B. Nothnick \\ Departments of Obstetrics and Gynecology, and Molecular and Integrative Physiology, \\ University of Kansas Medical Center, 3901 Rainbow Blvd, Kansas City, KS 66160, USA
}

\begin{abstract}
Tissue inhibitor of metalloproteinase 1 (TIMP-1) is a multifunctional protein expressed in the ovary and uterus of females of several species including mice, rats and humans. Mice deficient in TIMP-1 protein display altered reproductive parameters, which include reduced serum progesterone concentrations and abnormal uterine morphology. As these abnormalities could lead to altered reproductive performance, the objective of this study was to examine whether TIMP-1 deficiency is associated with a reduction in the reproductive lifespan of female mice in which the TIMP-1 gene is disrupted. Wild-type $(n=50)$ and TIMP-1 null mice $(n=90)$ were mated with males of proven fertility from the same genotype for 12 months during which the number of litters delivered and the number of pups per litter were determined. Significantly fewer TIMP-1 null females achieved pregnancy (47 of 90
\end{abstract}

or $52 \%$ ) compared with the wild-type mice (39 of 50 or $78 \% ; P<0.05)$. TIMP-1-deficient female mice that achieved and maintained pregnancy had significantly fewer litters during the 12 months $(2.9 \pm 0.8$ versus $3.5 \pm 0.8 ; P<0.01)$ and significantly fewer pups per litter $(5.7 \pm 1.3$ versus $4.7 \pm 1.1 ; P<0.05)$ than did wild-type mice. Females of both genotypes produced two consecutive litters, after which significantly fewer TIMP-1 null mice became pregnant. Mating of females with males of proven fertility from the other genotype confirmed that these abnormalities were not due to the inability of TIMP-1 null males to produce offspring. These data indicate that the absence of TIMP-1 is associated with a reduction in the reproductive lifespan of female mice, which may be manifested at the ovary, uterus or both organs.

\section{Introduction}

Tissue inhibitors of metalloproteinases (TIMPs) are expressed in the uteri of females in a variety of species including humans (Hampton and Salamonsen, 1994; Rodgers et al., 1994; Zhang and Salamonsen, 1997), nonhuman primates (Brenner et al., 1996), sheep (Hampton et al., 1995), pigs (Menino et al., 1997) and mice (Waterhouse et al., 1993; Das et al., 1997). In both humans and nonhuman primates (Hampton and Salamonsen, 1994; Rodgers et al., 1994; Brenner et al., 1996; Zhang and Salamonsen, 1997), TIMPs are postulated to control tissue remodelling and shedding of the endometrium during the menstrual period. However, TIMPs are also expressed beyond the period of menses within the uterus of these primate species, as well as within the uterus of females that do not show a period of menses (Waterhouse et al., 1993; Hampton and Salamonsen, 1994; Rodgers et al., 1994; Hampton et al., 1995; Brenner et al., 1996; Das et al., 1997; Menino et al., 1997). As TIMPs are multifunctional proteins (for a review, see Gomez et al., 1997), they could control a variety of cell functions that may play paramount roles in uterine physiology. For example, TIMP-1 has been shown to

Email:wnothnic@kumc.edu regulate cell proliferation (Bertaux et al., 1991; Hayakawa et al., 1992, 1994), programmed cell death (Alexander et al., 1996; Guedez et al., 1998), angiogenesis (Tagikawa et al., 1990; Johnson et al., 1994) and steroidogenesis (Boujard et al., 1995; Nothnick et al., 1997; Nothnick, 2000). Maintenance of a balance between cell proliferation and cell death, as well as uterine angiogenesis, and the role of the ovarian steroids within the uterus for the establishment of a successful pregnancy are essential. As such, TIMP-1 may contribute to the reproductive processes at a variety of cellular or biochemical levels.

Recent studies using TIMP-1-deficient mice have indicated that TIMP may play an important role in the reproductive process. TIMP-1 null mice have altered oestrous cycles, uterine morphology and systemic steroid concentrations (Nothnick, 2000). Alterations to these parameters could lead to reproductive difficulties. The aim of the present study was to determine whether the absence of TIMP-1 affects the ability of female mice to achieve pregnancy. Female mice that were unable to express a functional TIMP-1 gene product (that is, TIMP-1 knockout) were used to evaluate the effect of TIMP-1 deficiency on the long-term reproductive potential in TIMP-1 null female mice. Wild-type and TIMP-1 null mice were mated over 12 months, and the number of litters and pups per litter were calculated for each genotype during this period. 


\section{Materials and Methods}

\section{Animals}

TIMP-1 null and wild-type mice were used in all studies. TIMP-1-deficient mice (SVter 129 background) were generated by homologous recombination of a neo-containing gene-targeting vector in mouse embryonic stem cells. Transmission of the mutant allele and the genotype of mice were determined by PCR analysis of the neo sequences in genomic tail DNA. TIMP-1 deficiency was confirmed at the transcription and protein level by northern blot analysis and protease inhibitor assays, respectively (Nothnick et al., 1997).

Mice were housed within environmentally controlled conditions under the supervision of a licensed veterinarian. All animal procedures for these experiments were approved by the University of Kansas Medical Center Institutional Animal Care and Use Committee. Mice were maintained on a $14 \mathrm{~h}$ light:10 h dark cycle and were provided with water and mice chow ad libitum.

Female mice of each genotype ( $n=50$ wild-type and $n=90$ null mice, aged $7-8$ weeks) were used and all mice were fully inbred. Two to four littermates were selected randomly from each of 20 wild-type and 35 null litters. Females were mated with males of proven fertility of the same genotype and were checked twice a week for pregnancy. Pregnant females were removed and housed in separate cages. Females that did not become pregnant within 30 days after initial exposure to the males were excluded from further study and considered infertile. Pregnant females were allowed to nurse their pups for 21 days after which the pups were weaned and used for other experiments not related to this study. Once the pups were weaned, females were mated again with males of proven fertility of the same genotype and the procedure described above was repeated. This mating schedule continued until female mice failed to achieve pregnancy within 30 days of exposure to the males or until five consecutive litters were delivered. A period of approximately 12 months was termed the 'reproductive lifespan' of female mice. The percentage of fertile wild-type and TIMP-1 null mice (those that became pregnant within 30 days of exposure to the male) was calculated, in addition to the average number of litters per lifespan and the number of pups per lifespan. In addition, the number of pups born in each litter and the percentage of females producing pups with each consecutive litter were calculated between genotypes.

A separate study was conducted using 20 wild-type and 20 TIMP-1 null female mice to verify that any changes in reproductive lifespan were due to absence of the TIMP-1 gene product in the females (and not in the males). All of the females had previously delivered two litters and for the third mating, females were mated to males of proven fertility of the other genotype. The number of females that became pregnant and the number of pups born in each litter were then calculated and compared between genotypes of the females. The third mating period was chosen to assess

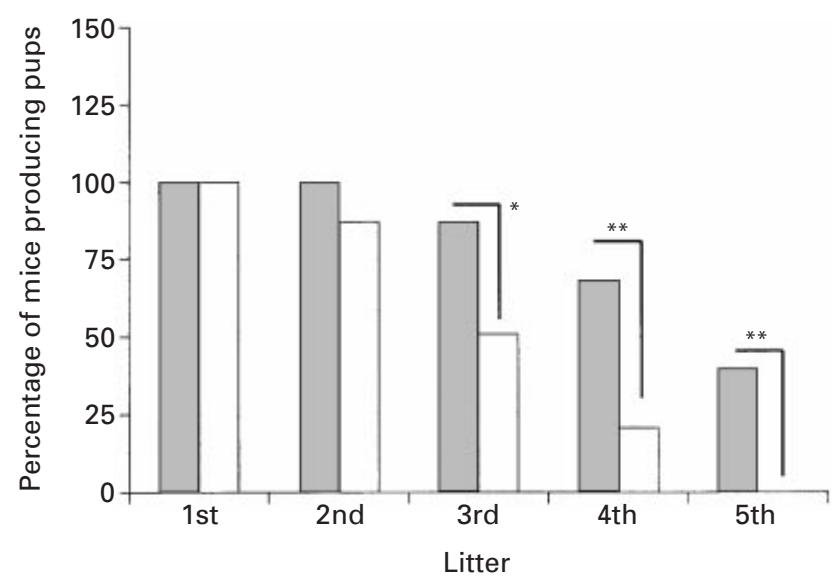

Fig. 1. The percentage of female mice of each genotype producing pups in each litter. The percentage of female mice delivering pups for each consecutive litter was calculated from the 39 wild-type $(\square, n=39)$ and 47 tissue inhibitor of metalloproteinase 1(TIMP-1) null mice $(\square, n=47)$ that became pregnant and delivered pups after the first mating. Asterisks indicate significant differences within each litter between wild-type and null mice using Fisher's exact test: ${ }^{*} P<0.05 ; * * P 0.005$.

reproductive performance of the females on the basis of the outcome of the first experiment, which demonstrated that there was a significant reduction in the percentage of null females achieving pregnancy from the third mating.

\section{Statistical analysis}

The percentages of female mice producing pups with each litter were compared within the litter number between genotypes using the Fisher's exact test. The number of pups per litter, the number of litters per lifespan and the number of pups per lifespan were compared using the unpaired $t$ tests. A comparison of the number of pups per litter within genotype across the number of litters born was made using one-way ANOVA. When a significant $F$ value was obtained, the Student-Newman-Keuls test was used for post-hoc analysis. Significance was set at $P<0.05$ for all studies and all data are displayed as the mean \pm the standard deviation (SD) from the mean.

\section{Results}

Within the first 30 days of exposure to males of proven fertility of the same genotype, significantly more wild-type females became pregnant (39 of 50 or $78 \%$ ) compared with TIMP-1 null females (47 of 90 or $52 \% ; P<0.05$ ). Wild-type females that became pregnant after the first mating and produced offspring produced up to five litters within 12 months. In particular, 100\% of wild-type females produced offspring after the first two matings, but this decreased to $87 \%, 68 \%$ and $40 \%$ for the third, fourth and fifth matings, respectively (Fig. 1). Similarly, most TIMP-1-deficient mice produced offspring after the first two matings; however, 


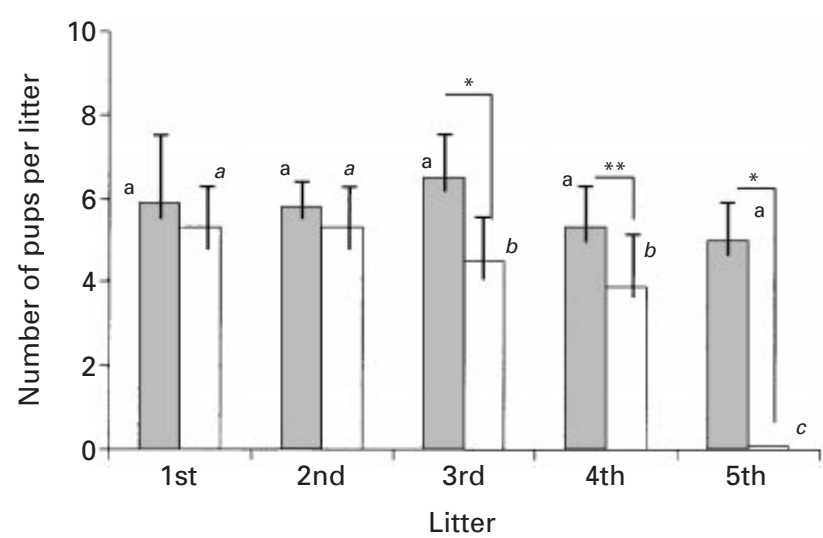

Fig. 2. Number of pups born per litter for each genotype. The average number of pups born with each consecutive litter was recorded for wild-type $(\square)$ and tissue inhibitor of metalloproteinase 1 (TIMP-1) null ( $\square$ ) mice. Data were obtained from 39 wild-type mice and 47 TIMP-1 null mice and are displayed as mean \pm SD. Different letters indicate significant differences within each genotype across the number of litters $(a, b$ for comparison within wild-type mice and $a, b$ for comparison within TIMP-1 null mice) by one-way ANOVA. Asterisks indicate statistically significant differences within litter between genotypes using unpaired $t$ tests: $* P<0.001 ; * * P<0.01$.

compared with wild-type mice, significantly fewer TIMP-1 null mice produced offspring after the third, fourth and fifth matings (51\%, $21 \%$ and $0 \%$, respectively; Fig. 1 ).

A similar pattern was also observed when the number of pups born per litter was assessed. Both wild-type and TIMP1 null mice gave birth to a similar number of pups in each of the first two litters (Fig. 2). However, by the third litter, TIMP-1 null mice gave birth to significantly fewer pups $(4.5 \pm 1.2$ versus $6.5 \pm 1.2$ for wild-type mice; Fig 2 .). In addition, significantly fewer pups were born to TIMP-1 null mice in the fourth $(3.9 \pm 1.0$ versus $5.4 \pm 1.3$ for wild-type mice) and fifth litters $(0 \pm 0$ versus $5.0 \pm 0.9$ for wild-type mice; Fig. 2). Overall (from the first to fifth mating), TIMP-1 null mice had significantly fewer litters per lifespan $(2.9 \pm 0.78$ versus $3.5 \pm 0.76)$ and significantly fewer pups per lifespan $(14.5 \pm 4.7$ versus $21.5 \pm 7.6 ; \quad P<0.001)$ compared with wild-type mice.

Wild-type and TIMP-1 null female mice were mated with reproductively proven males of the other genotype to verify that the decrease in the reproductive lifespan (that is, the ability to achieve pregnancy and deliver pups) was not influenced by the genotype of the males (Fig. 3). Similar to the initial study in which male and female mice of the same genotype were mated, significantly more $(P<0.05)$ wildtype female mice became pregnant when mated with null males (16 of 20 or $80 \%$ ) compared with TIMP- 1 null females mated with wild-type males ( 9 of 20 or $45 \%$; Fig. 3a). These wild-type female mice also delivered more pups compared with TIMP-1 null females $(5.6 \pm 1.0$ versus $4.4 \pm 1.3$; Fig. 3b).

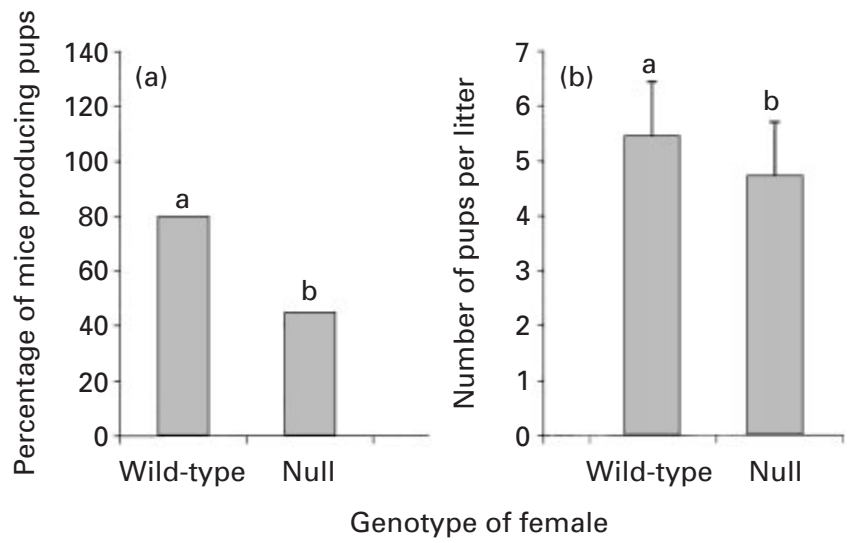

Fig. 3. The reduction in the reproductive lifespan of female tissue inhibitor of metalloproteinase 1 (TIMP-1) null mice is independent of the male genotype. Five- to six-month-old wild-type and TIMP-1 null female mice that had delivered two consecutive litters from previous matings with males of proven fertility from the same genotype were mated with males of proven fertility from the other genotype. Females were exposed to males for up to 30 days, after which they were excluded from the study. (a) The percentage of the wild-type and null females that delivered pups. (b) The number of pups born per litter resulting from mating of females and males of opposite genotypes. Different letters indicate significance by Fisher's exact test (a) and unpaired $t$ test (b).

\section{Discussion}

The present study demonstrates that TIMP-1 null female mice have fewer litters and fewer pups during their reproductive lifespan than do wild-type females. By the third mating between TIMP-1 null females and male mice, there was a significant reduction in the number of TIMP-1 null females that became pregnant. This reduction did not occur in TIMP-1 wild-type mice, indicating that TIMP-1 may either preserve the reproductive potential in these mice or that the absence of TIMP-1 is somehow deleterious to reproduction. Mating female and male mice to the other genotype indicated that the reduction in reproductive lifespan was attributed to the female, as significantly more wild-type females mated to TIMP-1 null males achieved pregnancy compared with null females mated to wild-type males. The processes affected by the absence of TIMP-1 could be at the ovary or uterus. Fewer TIMP-1 null mice achieved pregnancy, and of those that did significantly more failed to become pregnant with successive matings. Sharpe-Timms et al. (1998) demonstrated that women with altered fertility, such as women with endometriosis or unexplained infertility (K. Sharpe-Timms, personal communication) have significantly lower peritoneal fluid and serum concentrations of TIMP-1. These findings indicate that, in humans, TIMP-1 may play a role in the reproductive process and regulation of fertility. However, the mechanisms by which this is manifested are unknown.

Assessment of uterine morphology in TIMP-1 null mice of reproductive age indicated that there are marked 
alterations in the structure of the uterine lumen, which could influence implantation of the embryo (Nothnick, 2000). This alteration is associated with a significant increase in uterine matrix metalloproteinase (MMP) activity (W. Nothnick, unpublished), indicating that the absence of TIMP-1 leads to an imbalance in MMP activity. Increased expression of MMPs could influence a variety of biological functions within the uterus, which could have a negative effect on the reproductive process. Matrix metalloproteinases can regulate the bioavailability of cytokines (Gearing et al., 1994; Schönbeck et al., 1998) and growth factors (Fowlkes et al., 1994; Manes et al., 1997; Suzuki et al., 1997; Wu et al., 1999), which in turn can influence uterine cell proliferation (Shiraga et al., 1997; Zhang et al., 1998; Adesanya et al., 1999) or cell death (Tanaka et al., 1998; Lea et al., 1999). The observation that the effect of the absence of TIMP-1 on the reproductive lifespan of female mice does not occur until after the second litter indicates that prolonged absence of TIMP-1 (and prolonged increases in MMP activity) is required for these deficiencies to be manifested.

As progesterone is an essential steroid for the reproductive process, alterations in production or action of progesterone could reduce reproductive lifespan. TIMP-1 has been demonstrated to regulate progesterone production in vitro (Boujard et al., 1995) and TIMP-1-deficient mice of reproductive age have significantly lower concentrations of serum progesterone during oestrus, a time that coincides with the development of the early corpus luteum (Nothnick, 2000). Furthermore, subsequent studies using immature, gonadotrophin-primed TIMP-1 null mice confirmed the postulate that TIMP-1 null mice have reduced serum concentrations of progesterone during the period of corpus luteum development (W. Nothnick, unpublished). These significantly lower concentrations of progesterone may not be sufficient to allow for, or maintain, pregnancy in TIMP-1 null mice. As in the uterus, these alterations may be due to, and only become apparent after, prolonged absence of TIMP-1 (that is, after the birth of the second consecutive litter). In addition, improper periods or increased concentrations of cytokines resulting from the increased MMP activity could influence ovarian steroidogenesis. Cytokines, such as tumour necrosis factor $\alpha$ (Adashi et al., 1990; Benyo and Pate, 1992; Best et al., 1994) and interleukin $1 \beta$ (Best and Hill, 1995; Donesky et al., 1998; Kohen et al., 1999), suppress progesterone production in vitro and may influence progesterone production in vivo in TIMP-1 null mice.

In summary, the present study demonstrates that female TIMP-1 null mice have a shorter reproductive lifespan than do wild-type mice. This reduction may be due to insufficiencies at the uterus, ovary or both of the organs. Furthermore, insufficiencies may result from prolonged exposure to increased MMP activity, which occurs with the TIMP-1 null mice. Future studies will concentrate on determining the mechanisms and the level at which TIMP-1 influences the reproductive process in females.
This research was supported by a grant award (HD37941) from the Office of Research on Women's Health in conjunction with the $\mathrm{NICHD}$ and by NICHD/NIH through cooperative agreement U54 HD 33994 as part of the Specialized Cooperative Centers Program in Reproduction Research core support from NICHD center grant HD33994.

\section{References}

Adashi EY, Resnick CE, Puckman JN, Hurwitz Z and Payne DW (1990) Cytokine-mediated regulation of ovarian function: tumor necrosis factor alpha inhibits gonadotropin supported progesterone accumulation by differentiating and luteinized murine granulosa cells American Journal of Obstetrics and Gynecology 162 889-896

Adesanya OO, Zhou J, Samathanam C, Powell-Braxton L and Bondy CA (1999) Insulin-like growth factor-I is required for G2 progression in the estradiol-induced mitotic cycle Proceedings National Academy of Sciences USA 96 3287-3291

Alexander CM, Howard EW, Bissell MJ and Werb Z (1996) Rescue of mammary epithelial cell apoptosis and entactin degradation by a tissue inhibitor of metalloproteinases-1 transgene Journal of Cell Biology 135 1669-1677

Benyo DF and Pate JL (1992) Tumor necrosis factor-alpha alters bovine luteal cell synthetic capacity and viability Endocrinology 130 854-860

Bertaux B, Hornebeck W, Eisen AZ and Dubertret L (1991) Growth stimulation of human keratinocytes by tissue inhibitor of metalloproteinases Journal of Investigative Dermatology 97 679-685

Best CL and Hill JA (1995) Interleukin-1 alpha and -beta modulation of luteinized human granulosa cell oestrogen and progesterone biosynthesis Human Reproduction 10 3206-3210

Best CL, Pudney J, Anderson DJ and Hill JA (1994) Modulation of human granulosa cell steroid production in vitro by tumor necrosis factor alpha: implications of white blood cells in culture Obstetrics and Gynecology 84 121-127

Boujard N, Ogwuegbu SO, Garnier M, Lee CH, Martin BM and Papadopoulos V (1995) Identification of a stimulator of steroid hormone synthesis isolated from testis Science 268 1609-1612

Brenner RM, Rudolph L, Matrisian L and Slayden OD (1996) Non-human primate models; artificial menstrual cycles, endometrial matrix metalloproteinases and s.c. endometrial grafts Human Reproduction $\mathbf{1 1}$ 150-164

Das SK, Yano S, Wang J, Edwards DR, Nagase H and Dey SK (1997) Expression of matrix metalloproteinases and tissue inhibitors of metalloproteinases in the mouse uterus during the periimplantation period Developmental Genetics 21 44-54

Donesky BW, Dias de Moura M, Tedeschi C, Hurwitz A, Adashi EY and Payne DW (1998) Interleukin-1 $\beta$ inhibits steroidogenic bioactivity in cultured rat ovarian granulosa cells by stimulation of progesterone degradation and inhibition of estrogen formation Biology of Reproduction 58 1108-1116

Fowlkes JL, Enghild JJ, Suzuki K and Nagase H (1994) Matrix metalloproteinases degrade insulin-like growth factor-binding protein-3 in dermal fibroblast cultures Journal of Biological Chemistry 269 $25742-25746$

Gearing AJH, Beckett P, Christodoulou M et al. (1994) Processing of tumor necrosis factor- $\alpha$ precursor by metalloproteinases Nature 370 555-557

Gomez DE, Alonso DF, Yoshiji H and Thorgeirsson UP (1997) Tissue inhibitors of metalloproteinases: structure, regulation and biological functions European Journal of Cell Biology 74 111-122

Guedez L, Stetler-Stevenson WG, Wolff L, Wang J, Fukushima P, Mansoor A and Stetler-Stevenson M (1998) In vitro suppression of programmed cell death of B cells by tissue inhibitor of metalloproteinases-1 Journal of Clinical Investigation 102 2002-2010

Hampton AL and Salamonsen LA (1994) Expression of messenger ribonucleic acid encoding matrix metalloproteinases and their tissue inhibitors is related to menstruation Journal of Endocrinology 141 R1-R3 
Hampton AL, Butt AR, Riley SC and Salamonsen LA (1995) Tissue inhibitors of metalloproteinases in endometrium of ovariectomized steroid-treated ewes during the estrous cycle and early pregnancy Biology of Reproduction 53 302-311

Hayakawa T, Yamashita K, Tanzawa K, Uchijima E and Iwata K (1992) Growth-promoting activity of tissue inhibitor of metalloproteinase-1 (TIMP-1) for a wide range of cells. A possible new growth factor in serum FEBS Letters 298 29-32

Hayakawa T, Yamashita K, Ohuchi E and Shinagawa A (1994) Cell growthpromoting activity of tissue inhibitor of metalloproteinase-2 (TIMP-2) Journal of Cellular Science 107 2373-2379

Johnson MD, Kim HRC, Chesler L, Tsao-Wu G, Bouck N and Polverini PJ (1994) Inhibition of angiogenesis by tissue inhibitor of metalloproteinase Journal of Cell Physiology 160 194-202

Kohen P, Castro A, Caballero-Campo P, Castro O, Vega M, Makrigiannakis A, Simon C, Carvallo P and Devoto L (1999) Interleukin-1 beta (IL-1 $\beta$ ) is a modulator of human luteal cell steroidogenesis: localization of the IL type I system in the corpus luteum Journal of Clinical Endocrinology and Metabolism 84 4239-4245

Lea RG, Riley SC, Antipastis C, Hannah L, Ashworth CJ, Clark DA and Critchley HOD (1999) Cytokines and the regulation of apoptosis in reproductive tissues: a review American Journal of Reproductive Immunology 42 100-109

Manes S, Mira E, del Mar Barbacid M, Cipres A, Fernandez-Resa P, Buesa JM, Merida I, Aracil M, Marquez G and Martinez-AC (1997) Identification of insulin-like growth factor binding protein-1 as a potential physiological substrate for human stromelysin-3 Journal of Biological Chemistry 27225 706-25 712

Menino AR, Jr, Hogan A, Schultz GA, Novak S, Dixon W and Foxcroft GH (1997) Expression of proteinases and proteinase inhibitors during embryo-uterine contact in the pig Developmental Genetics 21 68-74

Nothnick WB (2000) Disruption of the tissue inhibitor of metalloproteinase1 gene results in altered reproductive cyclicity and uterine morphology in reproductive-age female mice Biology of Reproduction 63 905-912

Nothnick WB, Soloway PD and Curry TE, Jr (1997) Assessment of the role of tissue inhibitor of metalloproteinase-1 (TIMP-1) during the periovulatory period in female mice lacking a functional TIMP-1 gene Biology of Reproduction $\mathbf{5 6} 1181-1188$

Rodgers W, Matrisian LM, Giudice LC, Dsupin B, Cannon P, Svitek C, Gorstein F and Osteen KG (1994) Patterns of matrix metalloproteinase expression in cycling endometrium imply differential functions and regulation by steroid hormones Journal of Clinical Investigation 94 946-953

Schönbeck U, Mach F and Libby P (1998) Generation of biologically active
IL-1 $\beta$ by matrix metalloproteinases: a novel caspase-1-independent pathway of IL-1 $\beta$ processing Journal of Immunology 161 3340-3346

Sharpe-Timms KL, Keisler LW, McIntush EW and Keisler DH (1998) Tissue inhibitor of metalloproteinase- 1 concentrations are attenuated in peritoneal fluid and sera of women with endometriosis and restored in sera by gonadotropin-releasing hormone agonist therapy Fertility and Sterility 69 1128-1134

Shiraga M, Takahashi S, Miyake T, Takeuchi S and Fukamachi H (1997) Insulin-like growth factor-I stimulates proliferation of mouse uterine epithelial cells in primary culture Proceedings of the Society of Experimental Biology and Medicine 215 412-417

Suzuki M, Raab G, Moses MA, Fernandez CA and Klagsbrun M (1997) Matrix metalloproteinase-3 releases active heparin-binding EGF-like growth factor by cleavage at a specific juxtamembrane site Journal of Biological Chemistry 27231 730-31 737

Tagikawa M, Nishida Y, Suzuki F, Kishi J, Yamashita K and Hayakawa T (1990) Induction of angiogenesis in chick yolk-sac membrane by polyamines and its inhibition by tissue inhibitors of metalloproteinases (TIMP-1 and TIMP-2) Biochemical and Biophysical Research Communications 171 1264-1271

Tanaka T, Umesaki N, Mizuno K, Chang L, Ohtaki S and Ogita S (1998) Enhancement of apoptotic susceptibility by interleukin-1 beta in human endometrial epithelial cells Gynecologic Endocrinology 12 315-319

Waterhouse P, Denhardt DT and Khokha R (1993) Temporal expression of tissue inhibitors of metalloproteinases in mouse reproductive tissues during gestation Molecular Reproduction and Development 35 219-226

Wu H-B, Young Lee C and Rechler MM (1999) Proteolysis of insulin-like growth factor binding protein-3 in serum from pregnant, non-pregnant and fetal rats by matrix metalloproteinases and serine proteases Hormone and Metabolic Research 31 186-191

Zhang J and Salamonsen LA (1997) Tissue inhibitor of metalloproteinases (TIMP)-1, -2 and -3 in human endometrium during the menstrual cycle Molecular Human Reproduction 3 735-741

Zhang Z, Laping J, Glasser S, Day P and Hulholland J (1998) Mediators of estradiol-stimulated mitosis in the rat uterine luminal epithelium Endocrinology 139 961-966

Received 14 June 2001

First decision 27 July 2001.

Accepted 28 August 2001. 